\title{
NOTE ON LIE ALGEBRA KERNELS IN CHARACTERISTIC $p$
}

\section{G. HOCHSCHILD}

Let $K$ and $L$ be Lie algebras over a field $F$. Let $D(K)$ denote the derivation algebra of $K$, and $I(K)$ the ideal consisting of the inner derivations of $K$. If $\phi$ is a homomorphism of $L$ into $D(K) / I(K)$ then $\phi$ defines what is called the structure of an $L$-kernel on $K$. The $L$-kernel $K$ is said to be extendible if there exists a Lie algebra extension with kernel $K$ and image $L$ which induces the given $L$-kernel structure on $K$ in the natural fashion. The $L$-kernels with a fixed $L$-module $C$ as common center can be partitioned into equivalence classes, two kernels being equivalent if they differ (in the sense of a certain composition of kernels) by an extendible kernel. It has been shown in [2] that these equivalence classes of $L$-kernels constitute a vector group over $F$ which is canonically isomorphic with the 3-dimensional cohomology group $H^{3}(L, C)$. In particular, every $L$-kernel determines a 3-dimensional cohomology class which is called the obstruction of the kernel and whose vanishing is equivalent to the extendibility of the kernel.

A cohomology class $u$ for the finite dimensional Lie algebra $L$ in the finite dimensional $L$-module $C$ is said to be effaceable if there exists a finite dimensional $L$-module $C^{\prime}$ containing $C$ and such that the canonical image of $u$ in $H\left(L, C^{\prime}\right)$ is 0 . It is known from [2] and [3] that every effaceable 3-dimensional cohomology class for the finite dimensional Lie algebra $L$ in a finite dimensional $L$-module $C$ is the obstruction of a finite dimensional $L$-kernel $K$ with center $C$. Moreover, in the case of characteristic 0 , it has been shown in [3] that, conversely, the obstruction of a finite dimensional kernel is effaceable. The proof depends heavily on the structure and representation theory of Lie algebras of characteristic 0 and therefore breaks down completely in characteristic $p \neq 0$. The purpose of this note is to prove this result in the case of characteristic $p \neq 0$. When this is combined with the known results we have just mentioned there results the following theorem for arbitrary characteristic.

Theorem. Let $L$ be a finite dimensional Lie algebra and let $C$ be a finite dimensional L-module. Then an element of $H^{3}(L, C)$ is the obstruction of a finite dimensional L-kernel if and only if it is effaceable.

Received by the editors May 5, 1955. 
From now on all the Lie algebras and modules we shall consider will be assumed to be defined over a field $F$ of characteristic $p \neq 0$. Our principal tool is the universal enveloping algebra of a Lie algebra. The essential feature of our proof is that we augment a given $L$-kernel $K$ to a restricted $L^{*}$-kernel $K^{*}$, where $L^{*}$ and $K^{*}$ are finite dimensional restricted Lie algebras containing $L$ and $K$, respectively. The effaceability of the obstruction of $K$ will then follow from the fact that the obstruction of the $L^{*}$-kernel $K^{*}$ is effaceable. The construction of the restricted Lie algebras $K^{*}$ and $L^{*}$ will proceed within the universal enveloping algebras of $K$ and $L$, respectively, and will depend on the consideration of the $p$ th power map. Essentially, the technique we use in our construction is the same as that of Iwasawa [4] and Jacobson [5], although we are applying it here to a considerably more complicated situation.

For later reference, we collect a few well known facts concerning derivations of algebras of characteristic $p$.

(1) If $t$ is a derivation of a Lie algebra or of an associative algebra of characteristic $p$, then $t^{p}$ is also a derivation.

Let $U$ be either a Lie algebra or an associative algebra, and let $u \in U$. The inner derivation of $U$ which is effected by the element $u$ will always be denoted by $D_{u}$. If $U$ is an associative algebra we have $D_{u}(x)=u x-x u$. If $U$ is a Lie algebra we have $D_{u}(x)=[u, x]$.

(2) If $U$ is an associative algebra of characteristic $p$ and if $u \in U$ then the $p$ th power of the inner derivation effected by $u$ coincides with the inner derivation effected by $u^{p}$.

(3) Let $u$ and $v$ be elements of an associative algebra of characteristic $p$. Then $\sum_{t=0}^{p-1} u^{i} v u^{p-1-i}=D_{u}^{p-1}(v)$.

Now let $K$ be a Lie algebra over the field $F$ of characteristic $p$, and let $R_{K}$ denote the universal enveloping algebra of $K$. We define $P_{K}$ as the subspace of $R_{K}$ which is spanned by all elements of the form $x^{p^{n}}$, where $x \in K$ and $n=0,1, \cdots$ (for $n=0, x^{p^{n}}$ is to be interpreted as $x$ ).

Lemma 1. Let $t$ be a derivation of $R_{K}$ which maps $K$ into itself. Then $t\left(P_{K}\right) \subset K$.

Proof. We have to show that, for every $x \in K$ and every nonnegative integer $n$, we have $t\left(x^{p^{n}}\right) \in K$. For $n=0$, this holds by assumption. Generally, write $y$ for $x^{p^{n}}$. Using (3), we have $t\left(x^{p^{n+1}}\right)$ $=t\left(y^{p}\right)=\sum_{l=0}^{p-1} y^{i} t(y) y^{p-1-i}=D_{y}^{p-1}(t(y))$. By (2), we have $D_{y}=D_{x}^{p n}$, so that $t\left(x^{p^{n+1}}\right)=D_{x}^{p^{n}(p-1)}(t(y))$. The restriction of $D_{x}$ to $K$ is the inner derivation of the Lie algebra $K$ which is effected by the element $x \in K$. In particular, $D_{x}(K) \subset K$, whence our last result shows, by in- 
duction on $n$, that $t\left(x^{p^{n}}\right) \in K$, for all $x \in K$ and all $n$. This proves Lemma 1.

LEMMA 2. $P_{K}$ is closed under the commutation $[u, v]=u v-v u$ and the map $u \rightarrow u^{p}$, and thus is a restricted Lie algebra with these operations. Furthermore, if $\left(x_{i}\right)$ is a basis for $K$ over $F$, the elements $x_{i}^{p^{n}}(n=0,1, \cdots)$ constitute a basis for $P_{K}$ over $F$.

Proof. Let $y=x^{p^{n}}$, with $x \in K$. By (2), $D_{y}=D_{x}^{p^{n}}$ and therefore maps $K$ into itself. By Lemma 1 , we conclude that $D_{y}\left(P_{K}\right) \subset K$. Hence $P_{K}$ is closed under the commutation, and moreover we have $\left[P_{K}, P_{K}\right] \subset K$. Next we show that if $u \in P_{K}$ then also $u^{p} \in P_{K}$. This is evident from the definition of $P_{K}$ whenever $u$ is in $F x^{p^{n}}$, with some $x \in K$. Hence it suffices to prove that if $u, u^{p}, v, v^{p}$ are all in $P_{K}$ then also $(u+v)^{p} \in P_{K}$. For this purpose we recall that if $u$ and $v$ are elements of an associative algebra of characteristic $p$ one has $(u+v)^{p}$ $=u^{p}+v^{p}+s(u, v)$, where $s$, as a function of two independent noncommutative variables, is a certain sum of multiple commutators in these variables (a short proof of this result of Jacobson's will be found on p. 560 of [1]). In our case, since $u$ and $v$ are in $P_{K}$ and $\left[P_{K}, P_{K}\right] \subset K$, it follows therefore that $s(u, v) \in K$, whence $(u+v)^{p} \in P_{K}$. Finally, observe that this same argument shows that the subspace of $P_{K}$ which is spanned by the elements of the form $x_{i}^{p^{n}}$ is closed under the map $u \rightarrow u^{p}$, whence we conclude that it coincides with $P_{K}$. Since the elements $x_{i}^{p^{n}}$ are linearly independent, this proves the last assertion of Lemma 2.

Now let $L$ be a finite dimensional Lie algebra over $F$, and assume that $K$ is a finite dimensional $L$-kernel. Let $x \rightarrow t_{x}$ be a linear map of $L$ into the derivation algebra $D(K)$ of $K$ which induces the given kernel structure. This means that, for every $x \in L$, the derivation $t_{x}$ of $K$ belongs to the coset $\phi(x)$, where $\phi$ is the given homomorphism of $L$ into $D(K) / I(K)$. We can therefore find an alternating bilinear map $\tau$ of $(L, L)$ into $K$ such that, for all $x, x^{\prime}$ in $L,\left[t_{x}, t_{x^{\prime}}\right]=t_{\left[x, x^{\prime}\right]}+D_{\tau\left(x, x^{\prime}\right)}$.

We recall that every derivation of $K$ can be extended in one and only one way to a derivation of the associative algebra $R_{K}$. Moreover, an inner derivation $D_{x}$ of $K$ extends to the inner derivation of $R_{K}$ which is effected by $x$. It follows that no confusion can arise if we identify every derivation of $K$ with its canonical extension to a derivation of $R_{K}$. In order to keep our notation within reasonable bounds, we shall make this identification whenever it is convenient. In particular, it follows from the uniqueness of the extensions of derivations from $K$ to $R_{K}$ that the relation $\left[t_{x}, t_{x^{\prime}}\right]=t_{\left[x, x^{\prime}\right]}+D_{\tau\left(x, x^{\prime}\right)}$ still holds when these derivations are understood to be the extensions 
to $R_{K}$ of the original derivations of $K$. Moreover, when thus extended, each $t_{x}$ induces on $P_{K}$ a restricted derivation, with respect to the structure of $P_{K}$ as a restricted Lie algebra. Indeed, a derivation $t$ of a restricted Lie algebra $M$ with $p$-map $m \rightarrow m^{[p]}$ is said to be a restricted derivation if, for every $m \in M, t\left(m^{[p]}\right)=D_{m}^{p-1}(t(m))$; and we have already seen from (3) (at the beginning of our proof of Lemma 1) that if $t$ is any derivation of $R_{K}$ and $u \in R_{K}$ then $t\left(u^{p}\right)$ $=D_{u}^{p-1}(t(u))$.

If $u \in R_{K}$, we shall call a polynomial of the form $u^{p^{k}}+\gamma_{1} u^{p^{k-1}}$ $+\cdots+\gamma_{k} u$, where the $\gamma_{i} \in F$, and $k>0$, a $p$-polynomial in $u$. If $u \in K$ it follows from (2) that the inner derivation effected by any $p$-polynomial in $u$ maps $K$ into itself. It follows that, with $x_{1}, \cdots$, $x_{m}$ a basis for $K$ over $F$, we can find a $p$-polynomial $u_{i}$ in $x_{i}$ which commutes with every element of $K$ and therefore belongs to the center of $R_{K}$. Evidently, $u_{i} \in P_{K}$. Now let $z_{1}, \cdots, z_{n}$ be a basis for $L$ over $F$. Since, by Lemma $1, t_{z_{1}}$ maps $P_{K}$ into $K$ which is finite dimensional, there exists a $p$-polynomial $u_{i, 1}$ in $u_{i}$ which is annihilated by $t_{z_{1}}$. Clearly, $u_{i, 1}$ is also a $p$-polynomial in $x_{i}$. Next, there is a $p$-polynomial $u_{i, 2}$ in $u_{i, 1}$ which is annihilated by $t_{z_{2}}$, and also by $t_{z_{1}}$, of course. Continuing this construction, we finally obtain a $p$-polynomial $q_{i}=u_{i, n}$ in $x_{i}$ which is annihilated by each $t_{z_{j}}$, and hence also by each $t_{z}$ with $z \in L$. Let $Q_{K}$ denote the subspace of $P_{K}$ which is spanned by the elements $q_{i}^{p^{r}}, i=1, \cdots, m ; r=0,1, \cdots$. Evidently, $Q_{K}$ is a restricted ideal of $P_{K}$ and is contained in the center of $P_{K}$ (even of $R_{K}$ ). We put $K^{*}=P_{K} / Q_{K}$. Since our $p$-polynomials are all of degree greater than 1 , we have $Q_{K} \cap K=(0)$, so that we may identify $K$ with a Lie subalgebra of the restricted Lie algebra $K^{*}$. Furthermore, it is clear from our construction and the last assertion of Lemma 2 that $K^{*}$ is finite dimensional. It is also evident from our construction that the map $x \rightarrow t_{x}$ induces a linear map $x \rightarrow t_{x}^{*}$ of $L$ into the restricted derivation algebra of $K^{*}$ such that, for all $y, z$ in $L,\left[t_{y}^{*}, t_{z}^{*}\right]-t_{[y, z]}^{*}$ is the inner derivation of $K^{*}$ which is effected by the element $\tau(y, z) \in K$.

Now construct the restricted Lie algebra $P_{L}$. For our basis $z_{1}, \cdots$, $z_{n}$ of $L$, define $t^{*}\left(z_{j}^{p^{r}}\right)=\left(t_{z_{j}}^{*}\right)^{p^{r}}$, and extend this linearly to obtain a linear map $t^{*}$ of $P_{L}$ into the restricted derivation algebra of $K^{*}$. (In order to see that the $p$ th power of a restricted derivation is still a restricted derivation, note that, in the universal enveloping algebra, the property for a derivation $t$ to be restricted can be expressed by: $t\left(x^{[p]}\right)=t\left(x^{p}\right)$, where $x^{[p]}$ is the $p$-image of $x$ in the restricted Lie algebra and $x^{p}$ is the pth power of $x$ in the universal enveloping algebra). We claim that, for any $a$ and $b$ in $P_{L},\left[t^{*}(a), t^{*}(b)\right]-t^{*}([a, b])$ is an inner derivation of $K^{*}$, effected by an element of $K$. It is clearly 
sufficient to prove this in the case where $a$ and $b$ are of the form $z_{j}^{p^{r}}$. Our claim holds in virtue of what we have seen above when $r=0$, both in $a$ and in $b$. Making an induction on the degree, we may deduce the result to be proved as soon as we have shown that our claim holds for $a^{p}$ and $b$ whenever it holds for $a$ and $b$. Then we have $\left[t^{*}\left(a^{p}\right), t^{*}(b)\right]=\left[t^{*}(a)^{p}, t^{*}(b)\right]=D_{t^{*}(a)}^{p-1}\left(\left[t^{*}(a), t^{*}(b)\right]\right)$. If our claim holds for $a$ and $b$, this last derivation is $D_{i^{*}(a)}^{p-1}\left(t^{*}([a, b])+D_{x}\right)$, where $D_{x}$ denotes the inner derivation of $K^{*}$ which is effected by some element $x \in K$. Now $D_{t^{*}(a)}^{p-1}\left(D_{x}\right)=D_{y}$, where $y=t^{*}(a)^{p-1}(x)$. By definition, $t^{*}(a)$ maps $K$ into itself, whence $y \in K$. We have shown that $\left[t^{*}\left(a^{p}\right), t^{*}(b)\right]$ $=D_{t^{*}(a)}^{p-1}\left(t^{*}([a, b])\right)+D_{y}$, with some $y \in K$. If $a=z_{j}^{p^{r}}$ the first of the derivations on the right may be written in the form $D_{b^{*}\left(z_{j}\right)}^{p^{(}(-1)}\left(t^{*}([a, b])\right)$. From the beginning of our proof of Lemma 2, we know that $[a, b] \in L$. Hence we see that our derivation differs from $t^{*}\left(D_{z_{j}}^{p^{r}(p-1)}([a, b])\right)$ only by an inner derivation effected by an element of $K$. Since this last derivation is equal to $t^{*}\left(D_{a}^{p-1}([a, b])\right)=t^{*}\left(\left[a^{p}, b\right]\right)$, we conclude that our claim holds for $a^{p}$ and $b$. Thus we have indeed shown that $\left[t^{*}(a), t^{*}(b)\right]-t^{*}([a, b])$ is an inner derivation effected by an element of $K$, for all $a$ and $b$ in $P_{L}$.

Next, we shall show that, for every $a \in P_{L}$, the derivation $t^{*}(a)^{p}$ $-t^{*}\left(a^{p}\right)$ is also an inner derivation effected by an element of $K$. If $a \in F z_{j}^{p^{r}}$, this derivation is evidently 0 . Hence it suffices to show that if the result holds for $a$ and for $b$ it also holds for $a+b$. Now we observe that $t^{*}(a+b)^{p}-t^{*}\left((a+b)^{p}\right)=t^{*}(a)^{p}-t^{*}\left(a^{p}\right)+t^{*}(b)^{p}-t^{*}\left(b^{p}\right)$ $+s\left(t^{*}(a), t^{*}(b)\right)-t^{*}(s(a, b))$. Recalling that $s$ is a certain sum of multiple commutators, we may conclude from the result concerning commutators of derivations $t^{*}(a)$, etc., that the difference of the two terms involving $s$ is an inner derivation effected by an element of $K$. Hence our result holds for $a+b$ whenever it holds for $a$ and for $b$. This completes the proof of the second property of $t^{*}$.

In particular, it follows that $t^{*}$ induces a restricted homomorphism of the restricted Lie algebra $P_{L}$ into the restricted Lie algebra $D_{0}\left(K^{*}\right) / I\left(K^{*}\right)$, where $D_{0}\left(K^{*}\right)$ is the restricted derivation algebra of $K^{*}$ and $I\left(K^{*}\right)$ is the restricted ideal consisting of the inner derivations of $K^{*}$.

As before, using that $K^{*}$ and $L$ are finite dimensional, we can find a $p$-polynomial $r_{j}$ in $z_{j}$ which belongs to the center of $P_{L}$ and is such that $t^{*}\left(r_{j}\right)=0$. Let $Q_{L}$ denote the subspace of $P_{L}$ which is spanned by the elements $r_{j}^{p^{r}}, j=1, \cdots, n ; r=0,1, \cdots$. Then we see as before that $P_{L} / Q_{L}$ is a finite dimensional restricted Lie algebra $L^{*}$ and that $L$ may be identified with a subalgebra of $L^{*}$. Moreover, $t^{*}$ induces a linear map of $L^{*}$ into $D_{0}\left(K^{*}\right)$ (still denoted $t^{*}$ ) such that the deriva- 
tions $\left[t^{*}(a), t^{*}(b)\right]-t^{*}([a, b])$ and $t^{*}(a)^{p}-t^{*}\left(a^{p}\right)$ are inner derivations of $K^{*}$ effected by elements of $K$, for all $a$ and $b$ in $L^{*}$. In particular, if $a$ and $b$ are in $L$, then $\left[t^{*}(a), t^{*}(b)\right]-t^{*}([a, b])$ is the inner derivation of $K^{*}$ which is effected by the element $\tau(a, b)$ of $K$. Clearly, $t^{*}$ induces on $K^{*}$ the structure of a restricted $L^{*}$-kernel, in the sense of [2].

In order to be in a position to apply the cohomology theory of restricted Lie algebra kernels, we must make a slight modification of our kernel $K^{*}$. Let $C^{*}$ denote the center of $K^{*}$. Select any $p$-semilinear map $\rho$ of $K^{*}$ into $C^{* p}$ (i.e., for $b, c$ in $K^{*}$ and $\beta, \gamma$ in $F, \rho(\beta b+\gamma c)$ $\left.=\beta^{p} \rho(b)+\gamma^{p} \rho(c)\right)$ such that $\rho(c)=c^{p}$ whenever $c \in C^{*}$. Now define a new $p$-map $a \rightarrow a^{[p]}$ in $K^{*}$ by setting $a^{[p]}=a^{p}-\rho(a)$. It is well known and easy to check that this is still a legitimate $p$-map with which $K^{*}$ is a restricted Lie algebra. Furthermore, if a derivation of $K^{*}$ is a restricted derivation with respect to the original $p$-map $a \rightarrow a^{p}$ then it is also a restricted derivation with respect to the new $p$-map $a \rightarrow a^{[p]}$. For this new $p$-map we have $C^{*[p]}=(0)$, i.e., $C^{*}$ is strongly abelian. Hence $K^{*}$ has now been given the structure of a restricted $L^{*}$-kernel with strongly abelian center $C^{*}$, so that the cohomology theory of [2] applies to $K^{*}$.

We must now refer to pp. 709-713 of [2], where we have given a construction attaching to the restricted $L^{*}$-kernel $K^{*}$ with strongly abelian center $C^{*}$ a certain 3 -dimensional restricted cohomology class for $L^{*}$ in $C^{*}$. Since $L^{*}$ and $C^{*}$ are finite dimensional, this restricted cohomology class is effaceable. In fact, this follows easily from the definition of the restricted cohomology group (see [1]) noting that the restricted universal enveloping algebra of a finite dimensional restricted Lie algebra is finite dimensional. (Actually, if $U_{L^{*}}$ is the restricted universal enveloping algebra of $L^{*}$, our cohomology class is effaced in the module of all linear maps of $U_{L^{*}}$ into $C^{*}$ ).

Let $h$ be the canonical image in the ordinary cohomology group $H^{3}\left(L^{*}, C^{*}\right)$ of the restricted cohomology class attached to the restricted $L^{*}$-kernel $K^{*}$. Then $h$ is evidently still effaceable. Moreover, the construction we have referred to above shows that the cohomology class $h$ is represented by an alternating cocycle $f$ for $L^{*}$ in $C^{*}$ such that, for $x, y, z$ in $L, f(x, y, z)=t^{*}(x)(\tau(y, z))-t^{*}(y)(\tau(x, z))$ $+t^{*}(z)(\tau(x, y))-\tau([x, y], z)+\tau([x, z], y)-\tau([y, z], x)$ (see p. 713 of [2]). But this means precisely that the restriction of $f$ to $L$ (which actually takes values in $\left.C \subset C^{*}\right)$ represents the cohomology class $k$, say, in $H^{3}(L, C)$, which is the obstruction of our given $L$-kernel $K$, (see p. 702 of [2]). Hence the canonical image of $k$ in $H^{3}\left(L, C^{*}\right)$ coincides with the restriction of $h$ to $L$. Since $h$ is effaceable, so is its 
restriction to $L$. Hence the canonical image of $k$ in $H^{3}\left(L, C^{*}\right)$ is effaceable, whence also $k$ is effaceable. This completes the proof of our theorem.

\section{REFERENCES}

1. G. Hochschild, Cohomology of restricted Lie algebras, Amer. J. Math. vol. 76 (1954) pp. 555-580.

2. - Lie algebra kernels and cohomology, Amer. J. Math. vol. 76 (1954) pp. 698-716.

3. - Cohomology classes of finite type and finite dimensional kernels for Lie algebras, Amer. J. Math. vol. 76 (1954) pp. 763-778.

4. K. Iwasawa, On the representation of Lie algebras, Jap. J. Math. vol. 19 (1948) pp. $405-426$.

5. N. Jacobson, $A$ note on Lie algebras of characteristic p, Amer. J. Math. vol. 74 (1952) pp. 357-359.

UNIVERSITY OF ILLINOIS 